\title{
Chondrocalcinose de l'articulation temporo-mandibulaire : un cas
}

\author{
Nicolas Glock ${ }^{1}$, Jean-Marie Marteau ${ }^{1}$, Jean-Christophe Fricain ${ }^{1}$, \\ Laurent Hauret ${ }^{2}$, Sébastien Lepreux ${ }^{3}$
}

${ }^{1}$ UFR d'Odontologie, 146 rue Léo-Saignat, 33076 Bordeaux, France

${ }^{2}$ Cabinet de Radiologie, 7 allée de Chartres, 33000 Bordeaux, France

${ }^{3}$ Service d'Anatomie et Cytologie pathologiques, CHU, place Amélie Raba-Léon, 33076 Bordeaux, France

nicolasglock@hotmail.fr

La chondrocalcinose articulaire (CCA) a été décrite par Sitaj et Zitnan en 1957. La CCA est une maladie rhumatismale caractérisée par l'accumulation microcristaux de pyrophosphate de calcium dihydraté (PPCD) dans le cartilage articulaire (Khon et McCarthy 1962). La principale localisation de la CCA est le genou; les atteintes de l'ATM sont exceptionnelles. Cette pathologie est rarement évoquée dans le diagnostic différentiel des douleurs de l'ATM, en dehors des situations où la maladie a déjà été diagnostiquée dans une autre articulation.

Un cas clinique de chondrocalcinose de l'ATM est rapporté. Il s'agissait d'un homme de 58 ans présentant dans ces antécédents un rhumatisme articulaire aigu sans complication dans l'enfance, un kyste poplité au genou gauche en 1988, et une hernie discale opérée en 1996. Le motif de la consultation était une sensation d'oreille droite bouchée au retour des sports d'hiver. Il ne présentait pas d'otalgie, ni d'acouphène, ni de vertige, ni d'hyperthermie. L'ATM ne montrait pas de signe de déplacement du disque articulaire et il n'y avait pas d'antécédent traumatique. On notait l'absence de trismus et une ouverture buccale normale, non douloureuse. L'audiogramme était normal.

Un CT-scan sans injection révélait une zone d'ostéolyse bien limitée soufflant les corticales, avec des hyperdensités spumeuses dans la lésion. D'autres coupes montraient un contenu hyperdense dans l'interligne articulaire, sans apposition périostée. Cela orientait dans un premier temps le diagnostic vers une tumeur du rocher. Une IRM avec produit de contraste montrait un hypersignal dans la zone de la lésion mais aussi une coulée de prise de contraste vers l'ATM. Une du rocher de l'oreille droite révélait une tumeur développée à partir de la synoviale de l'ATM. On retrouvait des plages de tissu fibreux et cartilagineux sur lesquelles se détachaient de nombreuses calcifications arrondies et volumineuses. Au sein de ces calcifications, on notait la présence de plusieurs microcristaux quadrangulaires et biréfringents en lumière polarisée. En immunohistochimie, on observait une positivité du marqueur CD68, signant une réaction macrophagique. L'EMA qui marque les cellules épithéliales, le CD79a qui concerne les lymphocytes et les plasmocytes, et le CD45, qui est un marqueur pan-leucocytaire, étaient tous les trois négatifs; la protéine S 100 positive sur les cellules cartilagineuses. Le Mib1, qui marque les cellules engagées dans le cycle mitotique, était modérément élevé sur la composante histiocytaire.

L'aspect histologique confronté à l'aspect radiologique orientait le diagnostic vers une pseudo-goutte articulaire ou CCA de l'ATM (Faure et al. 1981, Hutton et al. 1987). Une ponction de liquide synovial afin de mettre en évidence les cristaux de PPCD pourrait confirmer le diagnostic. La colchicine est le traitement habituellement proposé (Alvarellos et al. 1986, Gonzalez et al. 1987) et l'oxaméthacine pour les crises aiguës. Le cortivazol (corticoïde à action prolongée) en infiltration a été aussi proposé (Bruce et al. 1997). 